\title{
Risankizumab: an anti-IL-23 antibody for the treatment of psoriasis
}

This article was published in the following Dove Press journal:

Drug Design, Development and Therapy

\author{
Isabel M Haugh' \\ Allie K Preston ${ }^{2}$ \\ Dario N Kivelevitch' \\ Alan M Menter' \\ 'Department of Dermatology, Baylor \\ University Medical Center, Dallas, \\ TX, USA; ${ }^{2}$ Texas A\&M Health Science \\ Center College of Medicine, Bryan, \\ TX, USA
}

\begin{abstract}
Risankizumab, a fully human IgG monoclonal antibody inhibitor of IL-23, is a therapeutic agent currently in late stage development for use in the treatment of moderate-tosevere plaque psoriasis. It is a biologic agent similar to guselkumab and tildrakizumab which targets IL-23 specifically, and has been primarily developed for use in moderate-to-severe psoriasis. USA-based pharmaceutical company Abbvie submitted it for a Biologics License Application to the US Food and Drug Administration (FDA) in April 2018. Risankizumab is the result of a collaboration between the German company Boehringer Ingelheim and Abbvie, which together are leading the future development and commercialization of risankizumab globally. The results from Phase I to Phase III clinical trials of risankizumab show it is highly effective and its FDA-approval in 2018 is likely. In this article we provide an independent expert opinion on the efficacy and safety of risankizumab in psoriasis based on a full review of the literature. Keywords: psoriasis, risankizumab, human monoclonal antibody, interleukin-23, efficacy, safety
\end{abstract}

\section{Introduction}

Risankizumab is a fully human IgG monoclonal antibody that binds with high affinity to the p19 sub-unit of IL-23 (IL-23A). ${ }^{1}$ IL-23 has two subunits, p19 and p40. The p19 subunit is specific to IL-23 whereas the p40 subunit is present on IL-12 in addition to IL-23. IL-23 plays a major role in controlling inflammation in peripheral tissues, particularly in type 1-polarized T cell immune responses. ${ }^{2}$ This is of particular relevance in psoriasis as it is known to be a T-cell-driven disease, type-1 cells are seen in excess numbers both in the lesional skin and peripheral circulation of psoriasis patients. ${ }^{3,4}$ The pathogenesis of psoriasis is multifactorial with the immune system, environmental factors, and genetics all playing major roles. Variants in the genes encoding the IL-23 receptor and the IL-23 p19 subunit (IL-23A) have been shown to be associated with potential for developing plaque psoriasis. ${ }^{1,5-7}$ IL-23 functions by triggering the differentiation of TH-17 and TH-22 cells which in turn trigger the inflammatory cascade responsible for the development of psoriatic lesions. ${ }^{1,7,8} \mathrm{TH}-17$ cells produce IL-17 which causes upregulation of a self-amplifying inflammatory response in keratinocytes within pre-psoriatic skin. IL-17 functions by recruiting leukocytes to psoriatic lesions and through induction of IL-19 and/or IL-36 activates STAT3, resulting in epidermal hyperplasia which results in plaque formation. ${ }^{9,10}$ Thus, by targeting IL-23, risankizumab inhibits the key inflammatory cascade which results in the production of IL-17.

\section{Phase I clinical study}

The Phase I double-blind, single-rising-dose, placebo-controlled clinical trial revealed excellent safety and efficacy of risankizumab in the treatment of plaque 
psoriasis. ${ }^{1}$ Thirty-nine patients aged between $18-75$ years with moderate-to-severe chronic plaque psoriasis for at least 6 months, a body surface area involvement of $10 \%$ or greater, a Psoriasis Area and Severity Index (PASI) score of 12 or greater, a Static Physician Global Assessment score of moderate or greater, and a body mass index of at least 18.5 but less than 40 were included in the study. ${ }^{1}$ Patients were randomized to receive a single dose of intravenous risankizumab, subcutaneous risankizumab or placebo. Twenty-four patients were assigned to the intravenous cohort and 15 to the subcutaneous cohort, thereafter, patients were assigned to one of seven sequential dose cohorts. In the first six cohorts, patients were randomly allocated to receive ascending single doses of risankizumab $(0.01,0.05,0.25,1,3$, or $5 \mathrm{mg} / \mathrm{kg})$ or matching placebo intravenously. ${ }^{1}$ Three patients per cohort received risankizumab and one received placebo. ${ }^{1}$ The seventh patient cohort was randomly allocated to receive one of two parallel doses, $0.25 \mathrm{mg} / \mathrm{kg}$ and $1 \mathrm{mg} / \mathrm{kg}$, of risankizumab or matching placebo subcutaneously. ${ }^{1}$ Of these, seven patients received $0.25 \mathrm{mg} / \mathrm{kg}$, six patients received $1 \mathrm{mg} / \mathrm{kg}$, and two patients received placebo subcutaneously. ${ }^{1}$

At week $12,87 \%, 58 \%$, and $16 \%$ of patients receiving risankizumab achieved a reduction in PASI score of $75 \%$, $90 \%$, and $100 \%$, respectively (Figure 1 ). ${ }^{1}$ In contradiction, no patient receiving placebo at week 12 had a reduction in PASI score of greater than $75 \%$. At 24 weeks, among patients receiving risankizumab intravenously or subcutaneously at doses of $0.25 \mathrm{mg} / \mathrm{kg}$ and greater $(\mathrm{n}=25), 84 \%, 60 \%$, and $36 \%$ achieved PASI scores 75,90 , and 100 , respectively. ${ }^{1}$
Decreases in PASI score were seen as early as week 2 after initiation of treatment. ${ }^{1}$ Risankizumab treatment was also associated with reduction in the expression of genes and proteins associated with the IL-17/IL-23 axis. ${ }^{1}$ Of interest, the gene expression profile of psoriatic lesions showed an important trend of normalization similar to that of nonlesional skin after treatment. ${ }^{1}$

In terms of safety, 24 weeks after initiation of treatment, $65 \%$ of patients $(20 / 31)$ receiving risankizumab intravenously or subcutaneously had an adverse event compared to $88 \%$ of patients $(7 / 8)$ receiving placebo. ${ }^{1}$ Of note, the severity of adverse events did not correlate with the dose of the risankizumab. ${ }^{1}$ Most commonly reported adverse events were upper respiratory infections, nasopharyngitis, and headache, side effects classically seen with all biologic agents for psoriasis. ${ }^{1}$ Four serious adverse events occurred in patients on risankizumab (alcoholic pancreatitis, ischemic thalamic stroke, transient ischemic attack, and polymyositis), but none were considered to be related to the study drug. ${ }^{1}$ Thus, the Phase I trial revealed that a single dose of risankizumab (intravenous or subcutaneous) is well-tolerated by the majority of patients and can effectively target the IL-23 pathway to induce substantial clinical improvements in patients with moderate-to-severe plaque psoriasis. ${ }^{1}$

Another anti-IL-23 antibody, ustekinumab, binds to the p40 subunit of IL-23 and thereby targets both IL-12 and IL-23. Ustekinumab has been shown to have significant efficacy and safety in the treatment of psoriasis. However, IL-23 is believed to play a more significant and critical role in the

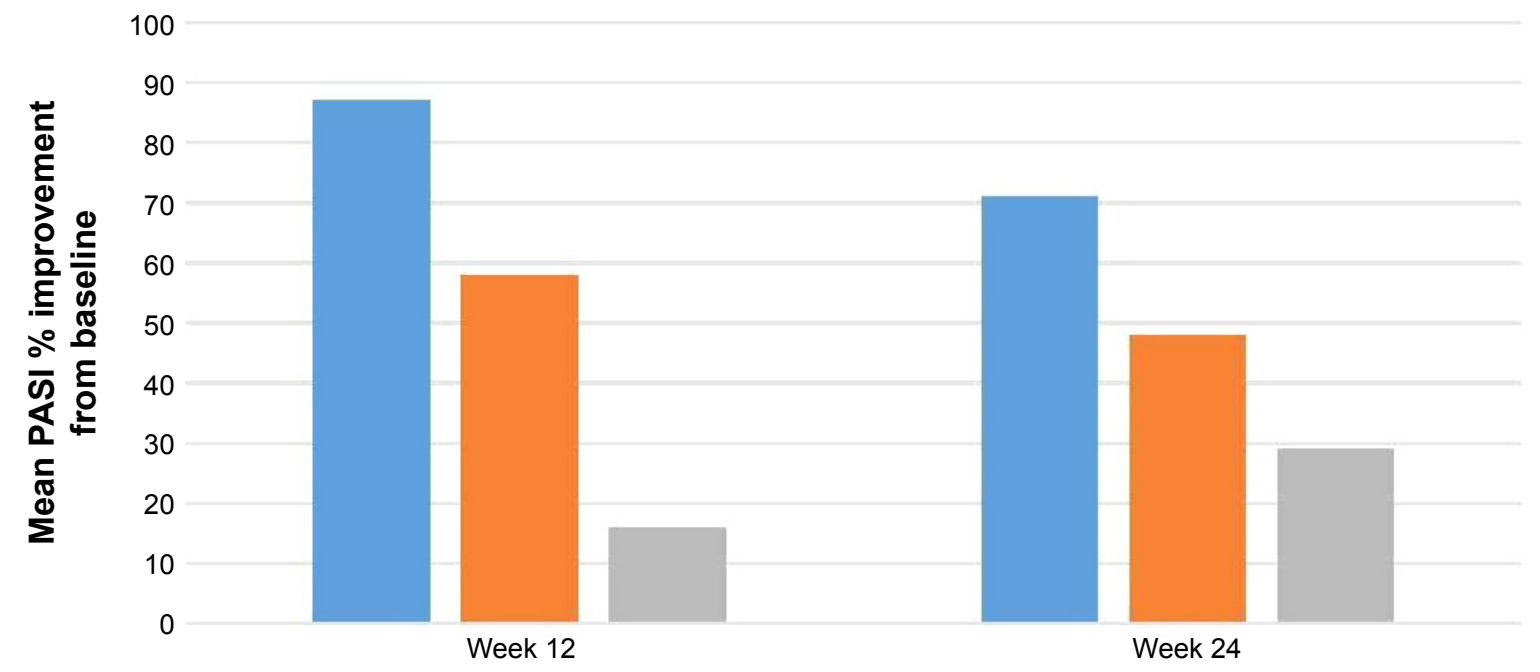

BI 655060 group

—PASI $75 \backsim$ PASI $90 \backsim$ PASI 100

Figure I Psoriasis Area and Severity Index (PASI) response rates in BI 655060 group of Phase I clinical trial. Note: Data from Krueger et al.' 
immunopathogenesis of psoriasis than IL-12. A comparison between risankizumab and ustekinumab was performed to test the relative efficacy between selective IL-23 inhibition via the p19 subunit and blockade of both IL-12 and IL-23 via the p40 subunit. ${ }^{12,13}$

\section{Phase II clinical study}

In the Phase II clinical comparison trial between risankizumab and ustekinumab, 166 patients with a PASI score of 12 or higher were randomly assigned to receive subcutaneous injections of risankizumab (a single $18 \mathrm{mg}$ dose at week 0 or $90 \mathrm{mg}$ or $180 \mathrm{mg}$ doses at weeks 0,4 , and 16) or ustekinumab (45 or $90 \mathrm{mg}$ based on weight greater or less than $100 \mathrm{~kg}$ at weeks 0,4 , and 16). ${ }^{12}$ At week 12, the percentage of patients with a $90 \%$ or greater reduction in PASI score was $77 \%$ in the risankizumab group and $40 \%$ in the ustekinumab group (Figure 2). ${ }^{12}$ At week 24, the percentage of patients who achieved at least $75 \%$ reduction in PASI score was $53 \%$ in the $18 \mathrm{mg}$ risankizumab group, $90 \%$ in the $90 \mathrm{mg}$ risankizumab group, and $88 \%$ in the $180 \mathrm{mg}$ risankizumab group, as compared with $70 \%$ in the ustekinumab group (Figure 2). Papp et al showed that improvements in PASI score were seen as early as week 2 in both the ustekinumab and risankizumab groups, with the onset of activity with risankizumab being faster and the duration of effect longer. ${ }^{12}$ Histopathologically, after 4 weeks, $54 \%$ and $69 \%$ of the patients in the $90 \mathrm{mg}$ and $180 \mathrm{mg}$ risankizumab groups, respectively, had assessments graded as indicating "excellent improvement", as compared with $29 \%$ of the patients in the ustekinumab group. ${ }^{12}$ Patient-reported outcomes in the study also revealed that risankizumab was more effective than ustekinumab in reducing joint pain in patients with psoriatic arthritis as well as improving scalp, palmoplantar, and fingernail psoriasis, all of which are associated with significant quality-of-life issues in these patients. ${ }^{12}$ At week $12,72 \%$ of patients treated with risankizumab 90 or $180 \mathrm{mg}$ achieved a Dermatology Life Quality Index score of 0 or 1, compared with 53\% treated with ustekinumab. ${ }^{12}$

This study was not large enough or long enough to adequately assess the safety of risankizumab, but risankizumab was shown to be well-tolerated and to have a safety profile similar to that of ustekinumab with no serious drug-related adverse events and no increase in drug-related events associated with increased dosages. ${ }^{12}$ Overall, these data suggest that selectively targeting the p19 subunit with risankizumab provides more complete blockade of IL-23 activity and greater efficacy in treating moderate-to-severe psoriasis than blockade of the p40 subunit with ustekinumab. ${ }^{7}$

Although both the Phase I and Phase II clinical trials of risankizumab have had relatively small sample sizes and short exposure times, the safety data to date have been reassuring. The most common adverse events were mild and not believed to be directly associated with risankizumab treatment. However, because of the lack of large-scale, long-term data thus far, the rate of uncommon adverse events and those events that may develop only after extended therapy are unknown. ${ }^{13}$ Regarding major adverse cardiac events (MACEs) which have been described with ustekinumab, ${ }^{14}$ in the Phase I study, cerebrovascular adverse events of ischemic thalamic stroke, and transient ischemic attack were reported in patients with

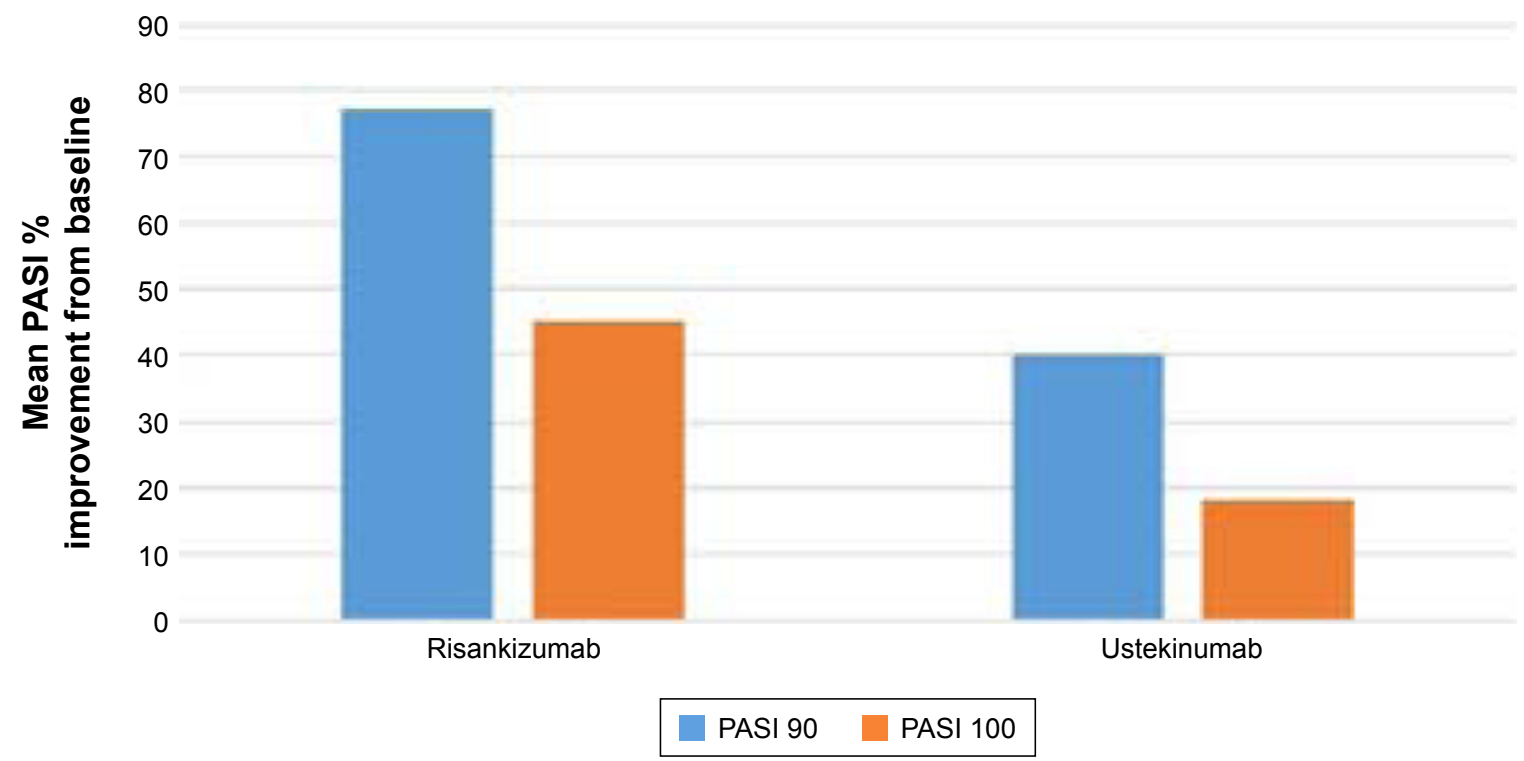

Figure 2 Psoriasis Area and Severity Index (PASI) response rates at week 12 in Phase II clinical trial. Note: Data from Papp et al. ${ }^{12}$ 
other pre-existing risk factors. ${ }^{13}$ In the Phase II trial, one patient with myocardial infarction and one patient with a cerebrovascular accident (on the day after elective surgery for a pre-existing cerebral aneurysm) were reported. ${ }^{13}$ It is well-known that cardiovascular disease in patients with moderate-to-severe psoriasis has a higher incidence than in patients without psoriasis, so it is quite possible that these MACEs were derived from the underlying disease process and not specifically related to the treatment itself. ${ }^{7}$ Additionally, risankizumab is being studied for use in the treatment of Crohn's disease, where higher therapeutic doses have been used as is the case with ustekinumab treatment for Crohn's disease. No dose-dependent association with adverse events has been detected to date. ${ }^{13}$

\section{Phase III clinical studies}

The Phase III clinical trial IMMhance examined the efficacy and safety of use of risankizumab in the treatment of chronic plaque psoriasis. ${ }^{15}$ The findings of the initial 16-week placebo-controlled period of this trial in patients with moderate-to-severe chronic plaque psoriasis revealed that at week 16, PASI 90 was achieved by $73.2 \%$ of the treatment group and $2.0 \%$ of placebo group (Figure 3). ${ }^{15}$ Three malignancies were reported in the risankizumab group and one MACE was reported in the placebo group. ${ }^{15}$ All events were considered as unlikely to be related to the study drug. ${ }^{15}$ Two Phase III clinical trials, UltIMMA-1 and UltIMMA-2, further investigated the use of risankizumab in this population. ${ }^{16}$ Patients were randomized to receive risankizumab $150 \mathrm{mg}$, ustekinumab 45 or $90 \mathrm{mg}$ (depending on weight) or placebo at weeks 0 and $4 .{ }^{16}$ At week 16 , the placebo group was switched to risankizumab, which was then administered to each group at weeks 16, 28, and 40. ${ }^{16}$ At week 16, in UltIMMA-1, PASI 90 was achieved by $75.3 \%$ of patients receiving risankizumab and $4.9 \%$ receiving placebo (Figure 3). ${ }^{16}$ In UltIMMA-2, these percentages were $74.8 \%$ and $2 \%$ respectively (Figure 3 ). ${ }^{16}$ Furthermore, at week 52, in UltIMMA-1, PASI 90 was achieved by $81.9 \%, 44.0 \%$, and $78.4 \%$ in the risankizumab, ustekinumab, and cross-over groups respectively. ${ }^{16}$ These percentages were $80.6 \%, 50.5 \%$, and $85.1 \%$ in the same groups in UltIMMA-2. ${ }^{16}$ In terms of safety, one patient was reported to have latent tuberculosis and one patient had seroconversion without latent disease. ${ }^{16}$ There was one sudden cardiac death and one myocardial infarction. ${ }^{16}$ All adverse events were considered non-study drug related. ${ }^{16}$ There was one death 161 days after the last dose of the study drug in the risankizumab group, a major adverse event considered to fall outside of the treatment-emergent window. ${ }^{16}$

\section{Conclusion}

The understanding of the crucial role of IL-17 and specifically IL-23 in the immunopathogenesis of psoriasis has led to a major change in our psoriasis therapeutic arsenal. The increased efficacy of IL-23 agents has made PASI 90 and PASI 100 the preponderant endpoints in clinical trials as compared to the prior PASI 50 and PASI 75 scores. Guselkumab, the initial FDA-approved anti-IL-23 agent has also shown impressive clinical efficacy and no new safety signals to date, with risankizumab showing similar efficacy in clinical trials. Nevertheless, long-term safety of risankizumab as well as other biologic agents in the IL-23 class is yet to be determined.

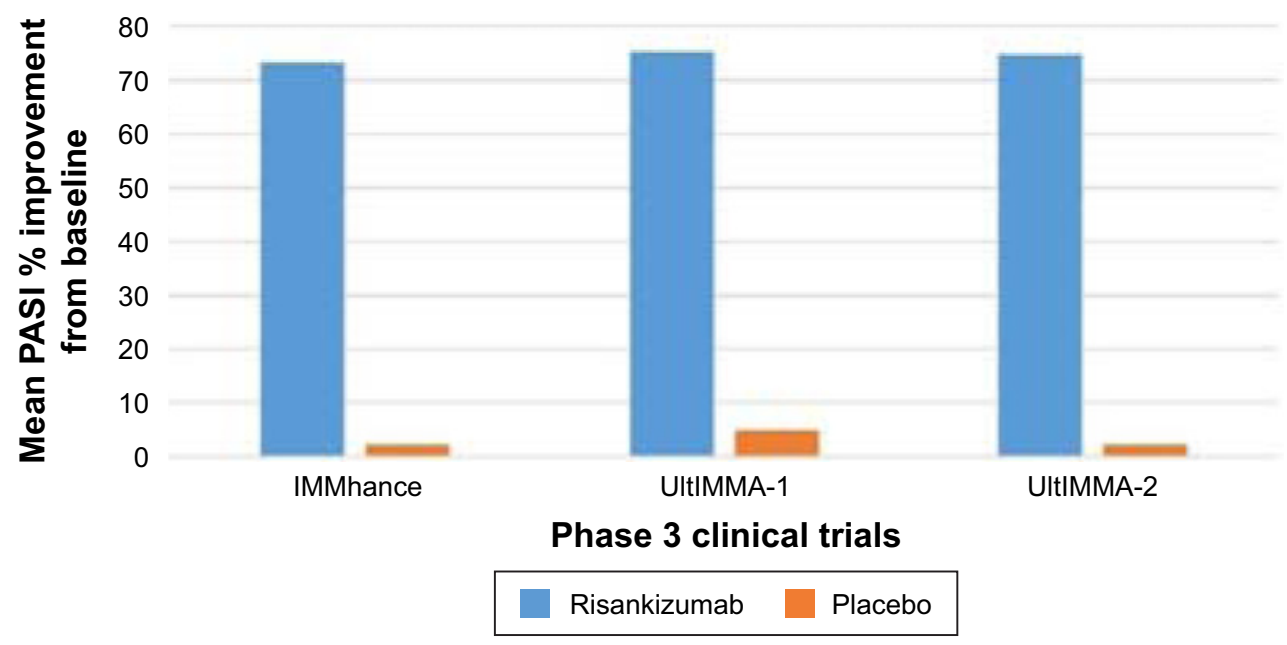

Figure 3 Psoriasis Area and Severity Index (PASI) 90 response rates at week 16 in Phase III clinical trial. Note: Data from Blauvelt et al ${ }^{15}$ and Gordon et al. ${ }^{16}$ 
Risankizumab is likely to be FDA-approved in 2018. It has all the characteristics to be an important and promising new biologic therapy for the treatment of moderate-to-severe plaque psoriasis, specifically due to its ability to limit the activity of the crucial IL-23/IL-17 inflammatory cascade.

\section{Disclosure}

Dr Menter is on the advisory board for AbbVie, Allergan, Amgen, Boehringer Ingelheim, Eli-Lilly, Janssen Biotech, Inc., and LEO Pharma; is a consultant for AbbVie, Allergan, Amgen, Eli-Lilly, Galderma, Janssen Biotech, Inc., LEO Pharma, Novartis, Pfizer, Vitae, and Xenoport; is an investigator for AbbVie, Allergan, Amgen, Anacor, Boehringer Ingelheim, Celgene, Dermira, Eli-Lilly, Janssen Biotech, Inc., LEO Pharma, Merck, Neothetics, Novartis, Pfizer, Regeneron, Symbio/Maruho, and Xenoport; is a speaker for AbbVie, Amgen, Janssen Biotech, Inc., and LEO Pharma; and has received compensation from AbbVie, Allergan, Amgen, Anacor, Boehringer Ingelheim, Celgene, Dermira, Eli-Lilly, Galderma, Janssen Biotech, Inc., LEO Pharma, Merck, Neothetics, Novartis, Pfizer, Regeneron, Symbio/ Maruho, Vitae, and Xenoport. The authors report no other conflicts of interest in this work.

\section{References}

1. Krueger JG, Ferris LK, Menter A, et al. Anti-IL-23A mAb BI 655066 for treatment of moderate-to-severe psoriasis: Safety, efficacy, pharmacokinetics, and biomarker results of a single-rising-dose, randomized, double-blind, placebo-controlled trial. J Allergy Clin Immunol. 2015;136(1):116-124.

2. Oppmann B, Lesley R, Blom B, et al. Novel p19 protein engages IL-12p40 to form a cytokine, IL-23, with biological activities similar as well as distinct from IL-12. Immunity. 2000;13(5):715-725.

3. Austin LM, Ozawa M, Kikuchi T, Walters IB, Krueger JG. The majority of epidermal T cells in Psoriasis vulgaris lesions can produce type 1 cytokines, interferon-gamma, interleukin-2, and tumor necrosis factor-alpha, defining TC1 (cytotoxic T lymphocyte) and TH1 effector populations: a type 1 differentiation bias is also measured in circulating blood T cells in psoriatic patients. J Invest Dermatol. 1999;113(5): 752-759.
4. Lee E, Trepicchio WL, Oestreicher JL, et al. Increased expression of interleukin $23 \mathrm{p} 19$ and $\mathrm{p} 40$ in lesional skin of patients with psoriasis vulgaris. $J$ Exp Med. 2004;199(1):125-130.

5. Cargill M, Schrodi SJ, Chang M, et al. A large-scale genetic association study confirms IL12B and leads to the identification of IL23R as psoriasis-risk genes. Am J Hum Genet. 2007;80(2):273-290.

6. Nair RP, Duffin KC, Helms C, et al. Genome-wide scan reveals association of psoriasis with IL-23 and NF-kappaB pathways. Nat Genet. 2009; 41(2):199-204.

7. Nestle FO, Kaplan DH, Barker J. Psoriasis. N Engl J Med. 2009;361(5): 496-509.

8. Lowes MA, Suárez-Fariñas M, Krueger JG. Immunology of psoriasis. Annu Rev Immunol. 2014;32:227-255.

9. Kim J, Krueger JG. Highly Effective New Treatments for Psoriasis Target the IL-23/Type 17 T Cell Autoimmune Axis. Annu Rev Med. 2017;68: $255-269$.

10. Harper EG, Guo C, Rizzo H, et al. Th17 cytokines stimulate CCL20 expression in keratinocytes in vitro and in vivo: implications for psoriasis pathogenesis. J Invest Dermatol. 2009;129(9):2175-2183.

11. Hawkes JE, Chan TC, Krueger JG. Psoriasis pathogenesis and the development of novel targeted immune therapies. J Allergy Clin Immunol. 2017; 140(3):645-653.

12. Papp KA, Blauvelt A, Bukhalo M, etal. Risankizumab versus Ustekinumab for Moderate-to-Severe Plaque Psoriasis. N Engl J Med. 2017;376(16): $1551-1560$.

13. Torres T. Selective Interleukin-23 p19 Inhibition: Another Game Changer in Psoriasis? Focus on Risankizumab. Drugs. 2017;77(14): $1493-1503$.

14. Ryan C, Leonardi CL, Krueger JG, et al. Association between biologic therapies for chronic plaque psoriasis and cardiovascular events: a metaanalysis of randomized controlled trials. JAMA. 2011;30(8):864-871.

15. Blauvelt A, Papp KA, Gooderham M, et al. Efficacy and Safety of Risankizumab, an IL-23 Inhibitor, in Patients with Moderate-to-Severe Chronic Plaque Psoriasis: 16-Week Results from the Phase 3 IMMhance Trial. Poster presented at: 14th Annual Maui Derm for Dermatologists Conference; January 28 to February 1; 2018; Maui.

16. Gordon KB, Strober B, Lebwohl M, et al. Efficacy and Safety of Risankizumab: Results from Two Double-Blind, Placebo- and UstekinumabControlled, Phase 3 Trials in Moderate-to-Severe Plaque Psoriasis. In: The American Academy of Dermatology Annual Meeting; February 16-20, 2018; San Diego, CA.

\section{Publish your work in this journal}

Drug Design, Development and Therapy is an international, peerreviewed open-access journal that spans the spectrum of drug design and development through to clinical applications. Clinical outcomes, patient safety, and programs for the development and effective, safe, and sustained use of medicines are the features of the journal, which

\section{Dovepress}

has also been accepted for indexing on PubMed Central. The manuscript management system is completely online and includes a very quick and fair peer-review system, which is all easy to use. Visit http://www.dovepress.com/testimonials.php to read real quotes from published authors. 